\title{
Extended Conformations of Polypeptides and Proteins in Urea and Guanidine Hydrochloride
}

\author{
M. LOIS TIFFANY and S. KRIMM, Biophysics Research Division, \\ Institute of Science and Technology, and Harrison M. Randall Laboratory \\ of Physics, University of Michigan, Ann Arbor, Michigan 48104
}

\section{Synopsis}

By analyzing the effect of urea and guanidine hydrochloride on the circular dichroism of many polypeptides and proteins, it is concluded that under conditions of high concentration of the perturbant and at low temperatures the resultant state approached is that of a local extended helix structure instead of a completely random coil. Intensification by urea and guanidine hydrochloride of the circular dichroism bands of poly-Lproline II leads to the proof that the mechanism of interaction of urea and guanidine hydrochloride with proteins is through hydrogen bonding to the backbone carbonyl group.

\section{INTRODUCTION}

Nemethy ${ }^{1}$ in 1967 stated that the mechanism of interaction of urea with proteins remains one of the unsolved problems of chemistry due to the difficulty of finding suitable techniques for demonstrating the involvement at the molecular level. It was once widely assumed that urea denatures proteins through an ability to break hydrogen bonds, ${ }^{2}$ but Schellman, ${ }^{3}$ in a study of the thermodynamics of the hydrogen bond in water, concluded that hydrogen bonds gave only marginal stability to ordered structures. Since the solubility in aqueous solution of many nonpolar compounds is increased by the addition of urea, Kauzmann ${ }^{4}$ suggested that urea contributes to the denaturation of proteins through a hydrophobic effect involving the side chains. By favoring exposure of the nonpolar group to the solvent, the hydrophobic interactions would be broken. Raising the temperature, substitution of alkyl groups for hydrogen in the urea molecule, and an increase in the length of the side chain in the substituted alkyl group would be expected to increase the effectiveness of denaturation by this mechanism. ${ }^{5}$ The possibility also exists of a direct involvement of the perturbant through binding to the backbone carbonyl or amide of the peptide chain. Thus Robinson and Jencks ${ }^{5}$ showed that the solubility of acetyltetraglycine ethyl ester increased linearly with concentration of urea or guanidine hydrochloride $(\mathrm{GuHCl})$ : $8 M$ urea increased the solubility 3.2-fold, while $7 M$ GuHCl increased it 7.4-fold. With both urea and $\mathrm{GuHCl}$ the solubility of the model compound increased on lowering the 
temperature from $40^{\circ} \mathrm{C}$ to $0^{\circ} \mathrm{C}$, and alkyl substitution decreased the solubilizing effectiveness. A final possible mode of action of urea (and of $\mathrm{GuHCl}$ ) on proteins may be through direct alteration of the solvent structure. $^{6}$

We have been interested in the conformations assumed by polypeptides and proteins in the presence of urea and $\mathrm{GuHCl}$, and have studied such systems by circular dichroism (CD). Our findings lead to the interpretation that these agents bind specifically to the peptide group, and also that they favor a locally ordered extended helix (EH) conformation rather than a "random" state of the chain. While the first conclusion is consistent with earlier ${ }^{5}$ as well as more recent ${ }^{7,8}$ speculations, the second represents a more detailed specification of chain conformation than has prevailed until now.

\section{EXPERIMENTAL AND RESULTS}

Circular dichroism spectra were recorded using a Sproul-modified Durrum Jasco Model 5 instrument.

Most polypeptides and proteins were studied at concentrations of $\mathbf{0 . 5}$ $\mathrm{gm} / \mathrm{l}$ in urea and $\mathrm{GuHCl}$ solutions, using a $1 \mathrm{~mm}$ temperature-controlled cell. The urea was Baker Analyzed Reagent Grade (Baker Chemical Co.) and the GuHCl was "ultrapure," obtained from Heico, Inc. The following results were obtained.

1. Poly-L-proline (Sigma, m.w. 13,500) shows a continuing increase in intensity of both bands characteristic of form II when up to $5.7 \mathrm{M} \mathrm{GuHCl}$ is added (the highest concentration used). A similar continuing increase in the positive band is recorded when urea is added, up to $9.5 M$ (Fig. 1).

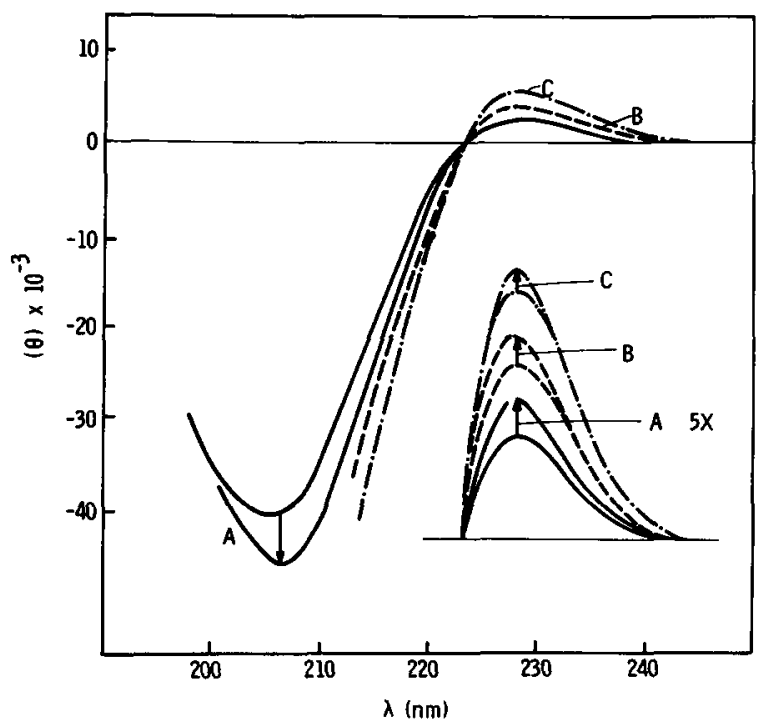

Fig. 1. Circular dichroism spectra of poly-L-proline in (A) water, (B) $9.5 M$ urea, (C) $5.7 \mathrm{M} \mathrm{GuHCl}$. The arrows indicate the effect of lowering the temperature from $25^{\circ} \mathrm{C}$ to $5^{\circ} \mathrm{C}$. 
2. Poly-L-lysine hydrochloride (Pilot) in the ionized form at $\mathrm{pH} 7$ shows a positive band at $218 \mathrm{~nm}$. This band continuously increases in intensity as urea is added (Fig. 2a). In $0.66 \mathrm{M} \mathrm{GuHCl}$ solution, on the other hand, samples at pH 7 show an initial decrease at $218 \mathrm{~nm}$ to about $3 / 4$ of the intensity in aqueous solution (Fig. 3a). This is followed, at $3.8 \mathrm{M} \mathrm{GuHCl}$, by a return of the band shape and intensity to those in aqueous solution; and, at $5.7 \mathrm{M} \mathrm{GuHCl}$, by a band intensity about $1 \frac{1}{4}$ times that in water (Fig. 3a). At pH 11 in 8 or $9 M$ urea, we again find a $218 \mathrm{~nm}$ positive band, after first disrupting the $\alpha$-helical form (Fig. 2b). Lowering the temperathe temperature increases the intensity of this band.
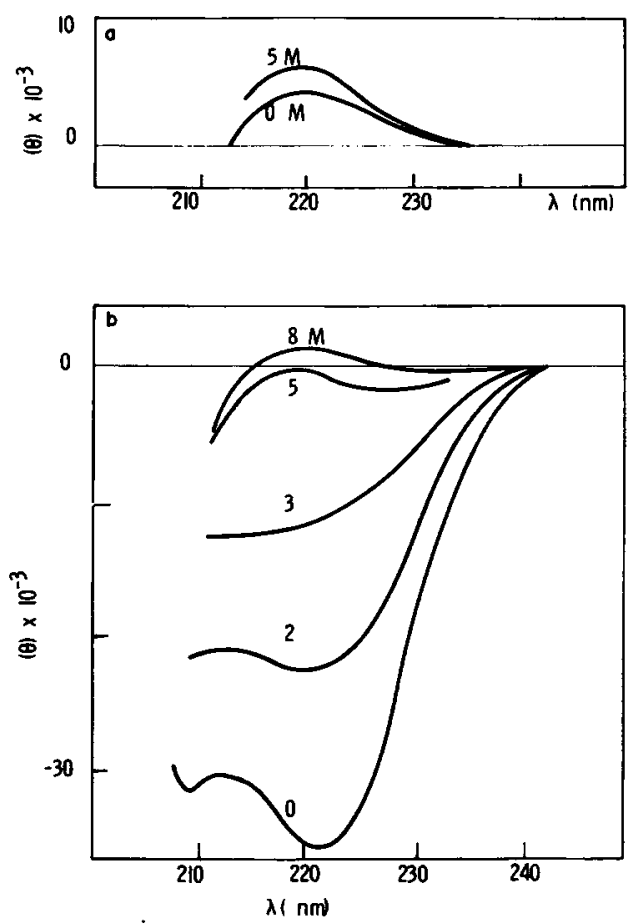

Fig. 2. Circular dichroism spectra of poly-L-lysine, (a) at pH 7 in $5 M$ urea at $25^{\circ} \mathrm{C}$, (b) at $\mathrm{pH} 11$ and $25^{\circ} \mathrm{C}$ in varying concentrations of urea.

3. Poly-L-glutamic acid (Pilot) in the ionized form at $\mathrm{pH} 7$ shows a positive band at $218 \mathrm{~nm}$. This band increases in intensity by lowering the temperature and by the addition of urea (Fig. 4). In $0.66 \mathrm{M} \mathrm{GuHCl}$ solution, samples at $\mathrm{pH} 7$ show an initial decrease at $218 \mathrm{~nm}$ to about $2 / 3$ of the intensity in aqueous solution (Fig. $3 \mathrm{~b}$ ). In $5.7 \mathrm{M} \mathrm{GuHCl}$ solution the intensity of this band increases, but only to about 0.8 of its value in water (Fig. $3 \mathrm{~b}$ ). The $\alpha$-helical state of the unionized polymer at pH 4.3 is partially upset by $8 M$ urea (Fig. 4$)$. When the $\mathrm{pH}$ is still lower $(\sim 2)$, so that the CD spectrum gives indication of a highly aggregated form, ${ }^{9}$ the addition of urea first removes signs of aggregation, so that the polymer actually appears to be more $\alpha$-helical (Fig. 5). 


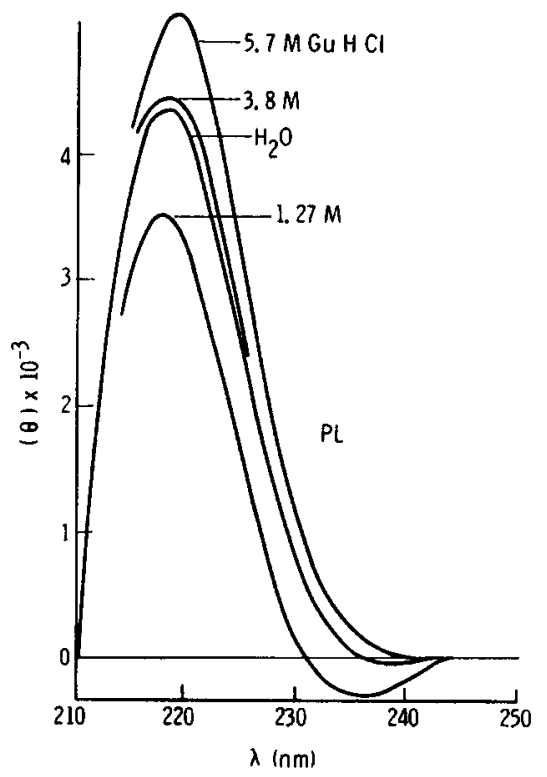

(a)

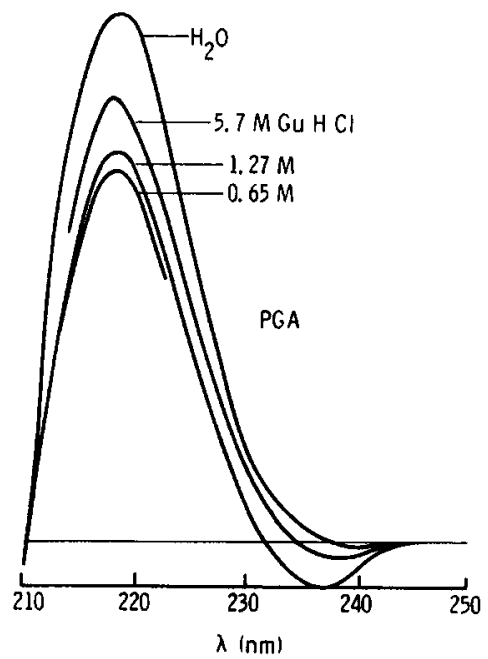

(b)

Fig. 3. Circular dichroism spectra of (a) ionized poly-L-lysine (pH 7) and (b) ionized poly-L-glutamic acid ( $\mathrm{pH} \mathrm{7)}$, as a function of $\mathrm{GuHCl}$ concentration.

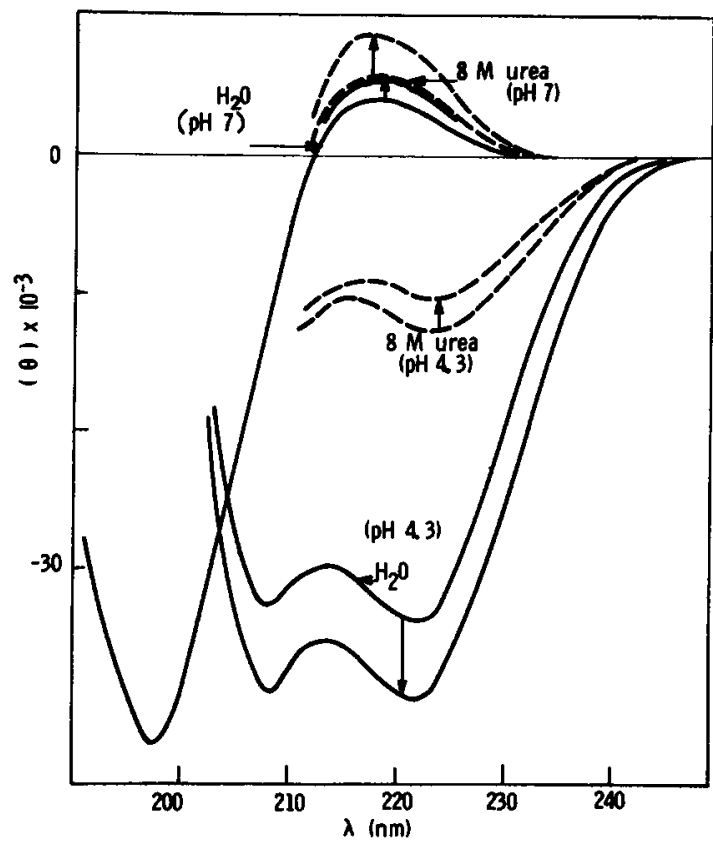

Fig. 4. Circular dichroism spectra of poly-L-glutamic acid at various pH's and urea concentrations. The arrows indicate the effect of lowering the temperature from $25^{\circ} \mathrm{C}$ to $5^{\circ} \mathrm{C}$. 


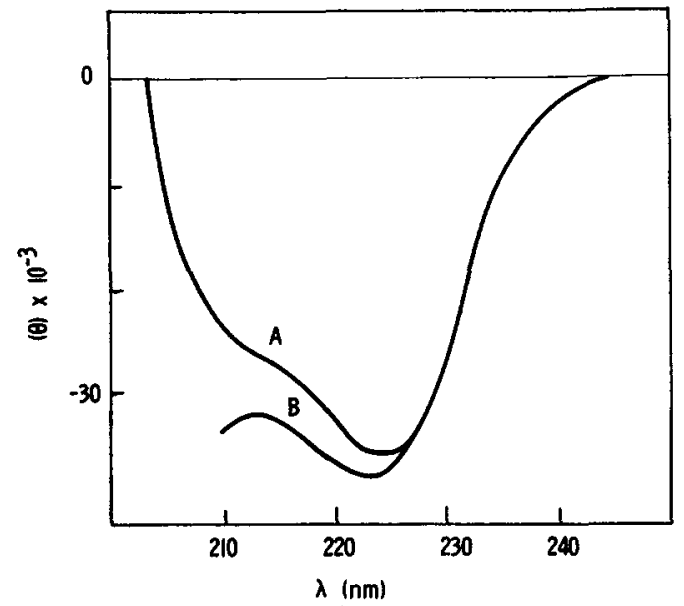

Fig. 5. Circular dichroism spectra of poly-L-glutamic acid, (A) at $\mathrm{pH} \sim 2$ in water, (B) at $\mathrm{pH} \sim 2$ in $5 M$ urea.

4. Poly- $N^{5}$-(3-hydroxypropyl)-L-glutamine (Pilot) has a negative CD at $220 \mathrm{~nm}$ in aqueous solution. In $8 M$ urea or in $6 M \mathrm{GuHCl}$ a strong positive CD band appears at this wavelength $([\theta]=4000)$, even at room temperature.

5. Bovine brain $\mathbf{A}_{1}$ protein ${ }^{10}$ (kindly provided by Dr. Eylar) shows a definite development of a positive band near $218 \mathrm{~nm}$ when dissolved in $8 M$ urea. The band increases in intensity on lowering the temperature (Fig. 6).

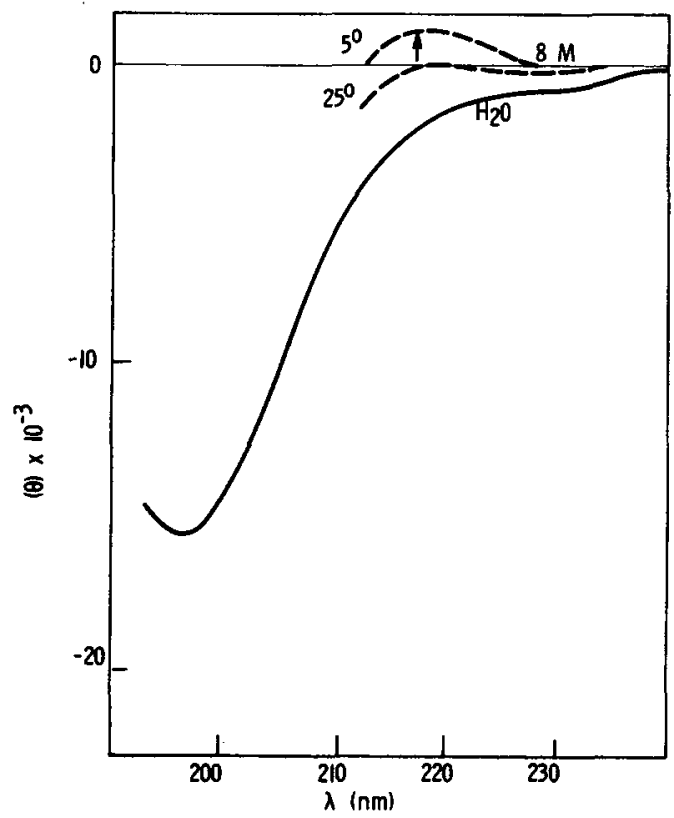

Fig. 6. Circular dichroism spectra of bovine brain $A_{1}$ protein, in water and in $8 M$ urea. 
6. Myoglobin, sperm whale (CAB), is resistant to urea denaturation. Apparently it is difficult to open up the molecule. But by heating a sample in $9 M$ urea to $95^{\circ} \mathrm{C}$ for 5 minutes and then cooling, a positive CD band is recorded in the $220 \mathrm{~nm}$ region (Fig. 7).

7. Ribonuclease A (Sigma) is easily denatured by $8 M$ urea. The final state as recorded by $\mathrm{CD}$ is very temperature-sensitive. At $5^{\circ} \mathrm{C}$ there is a definite indication in the spectrum of a positive band in the $220 \mathrm{~nm}$ region (Fig. 8). A similar band is produced by $5.7 M \mathrm{GuHCl}$ at $5^{\circ} \mathrm{C}$.

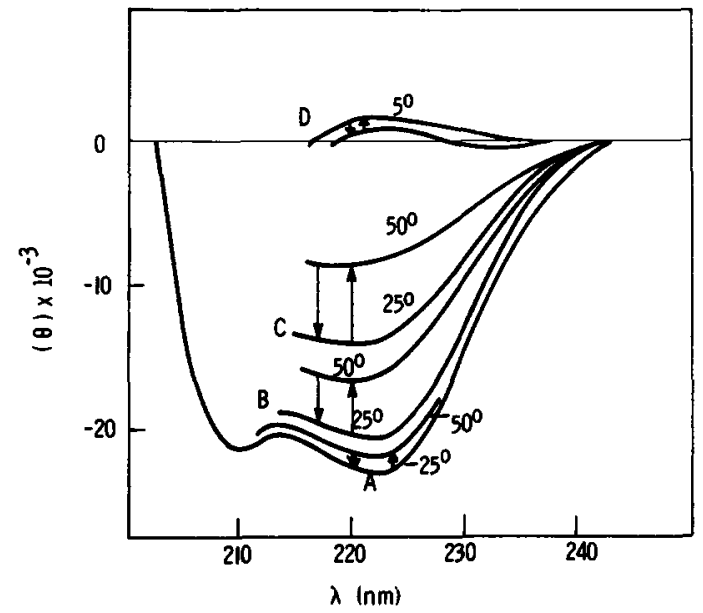

Fig. 7. Circular dichroism spectra of sperm whale myoglobin, (A) in water, (B) in $8 M$ urea, (C) in $9 M$ urea, (D) in $9 M$ urea, heated to $95^{\circ} \mathrm{C}$ for 5 min and then cooled (lower curve at $25^{\circ} \mathrm{C}$ ).

8. Lysozyme, hen egg white (Sigma) is not easily denatured by urea. Many recent studies have been done on the effect of urea and GuHCl. ${ }^{11-14}$ The initial increase in intensity of the positive bands in the $290-\mathrm{nm}$ region by urea and GuHCl (Fig. 9) also occurs under other conditions, such as low alcohol and denaturing salts. ${ }^{15}$ All near-u.v. bands are seen to disappear upon heating in urea plus reducing agent (Fig. 9). Disruption of the far-u.v. CD occurs in $4.75 M \mathrm{GuHCl}$ with no reducing agent, but a small negative band is left in the near-u.v. region at $275 \mathrm{~nm}$ (probably due to the S-S bond). A shoulder appears in the $220 \mathrm{~nm}$ region but is not pronounced.

Other proteins tested, including $\beta$-lactoglobulin, insulin, and pepsin, show only a high shoulder in the 220 -nm region in their CD spectra in $9 M$ urea or $6 M \mathrm{GuHCl}$, even at low temperatures.

In the above studies we have made no attempt to correct for the index of refraction of the solvent. We do this because Goodman and co-workers ${ }^{16}$ have reported little difference in the ORD of rigid bicyclic lactams in water or in $8 M$ urea. 


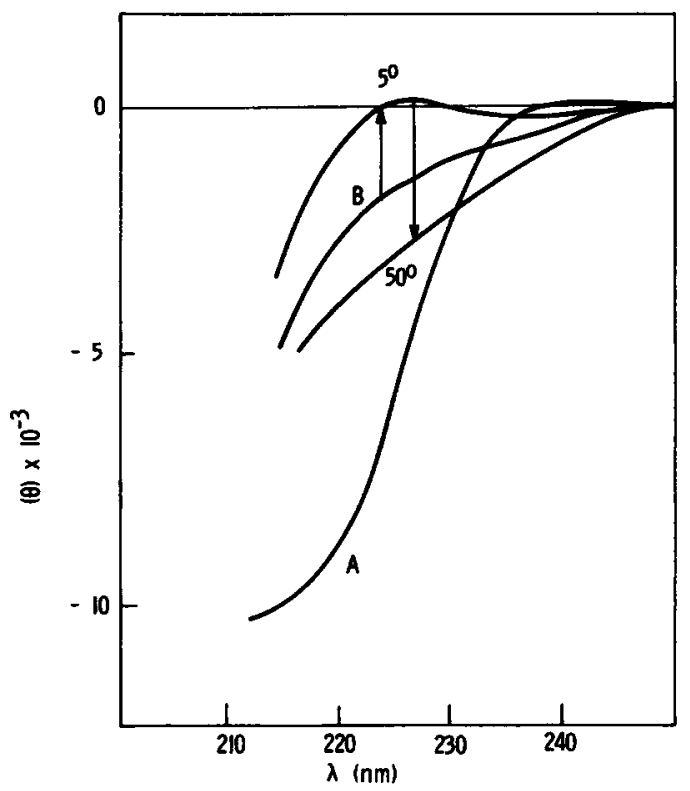

Fig. 8. Circular dichroism spectra of ribonuclease $A,(A)$ in water, (B) in $8 M$ urea at $25^{\circ} \mathrm{C}$ cooled to $5^{\circ} \mathrm{C}$, and warmed to $50^{\circ} \mathrm{C}$.

\section{DISCUSSION}

An understanding of the above results depends on a proper assignment of the observed CD spectra. This in turn is related to our understanding of the nature of the "random" state of a polypeptide chain. A reasonable definition is that it comprises those "... spatial configurations which have

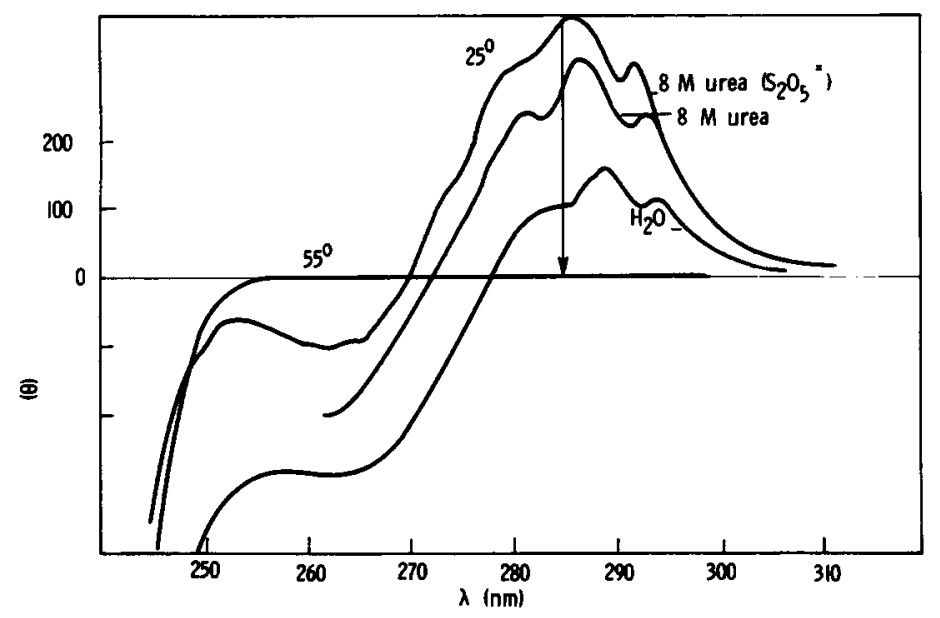

Fig. 9. Circular dichroism spectra of hen egg white lysozyme; in water, in $8 M$ urea with and without reducing agent, and the latter sample heated to $55^{\circ} \mathrm{C}$ (note disappearance of bands in 255 to $310 \mathrm{~nm}$ region). 
no recurrent pattern of replication of bond conformations along the chain...."17 Thus, in the case of a polypeptide chain the allowed regions of the $\varphi, \psi$ map are sampled statistically, conformations being present according to a Boltzmann weighting of the relative energies. In the past it had been tacitly assumed that these corresponded to a typical dipeptide energy map. ${ }^{18}$ In addition, the CD spectrum of a random coil was equated with that of charged poly-L-lysine (PL) or poly-L-glutamic acid ${ }^{19}$ (PGA).

That the above two propositions are basically contradictory was pointed out by us in a series of papers..$^{20-25}$ In the first place, it was noted ${ }^{20-23}$ that the CD spectra of systems such as heat-denatured collagen, which were expected to be completely unordered, were not the same as the spectra of charged PL and PGA. A wide variety of polypeptides and proteins under conditions where they would be expected to be unordered gave spectra qualitatively similar to that of heat-denatured collagen, ${ }^{23}$ viz., a single negative band near $200 \mathrm{~nm}$. Secondly, it was shown ${ }^{24,25}$ that the conformational energy map for polypeptides with charged side chains is expected to be different from the map for uncharged residues. This provided the basis for understanding the fact that the PL and PGA spectra were different from those of the more unordered systems, viz., being characterized by a weak positive band near $220 \mathrm{~nm}$ as well as a strong negative band near $200 \mathrm{~nm}$. The energy minima resulting from side-chain electrostatic repulsions were found to occur in the region of the threefold lefthanded helix ${ }^{24}$ and this locally extended structure was called the extended helix $(\mathrm{EH})$ conformation. ${ }^{23}$

Significant evidence has since accumulated to support the above ideas: The CD spectrum of poly (ala gly gly), a polypeptide which was shown to be in the EH conformation, is found to be similar to that of charged PL and PGA. ${ }^{26}$ Polypeptides which, on the basis of their CD spectra, would nominally be assigned a local EH conformation, exhibit a temperature dependence which is more consistent with such an assignment than with an assignment to a mostly unordered state. ${ }^{27}$ A theoretical calculation of the CD spectrum of an unordered chain, ${ }^{28}$ based on a standard dipeptide energy map,$^{18}$ in fact predicts a spectrum qualitatively more similar to our assignment $^{23}$ than to that observed for charged PL and PGA. Finally, additional theoretical calculations ${ }^{29}$ confirm our prediction ${ }^{24}$ of the restricted region of the $\varphi, \psi$ map associated with polypeptides containing charged side chains.

In summary, we must expect ${ }^{27}$ that at least two kinds of conformational states lacking long-range order can exist: one, the unordered state, represented by a wide sampling of a standard dipeptide energy map, ${ }^{18}$ and possessing the lowest degree of regularity; and two, the EH state, represented by a narrow sampling of a smaller region of the $\varphi, \psi$ map. ${ }^{2}$. Although the latter situation does not yield the long-range regularity of, say, the $\alpha$-helix, it does give rise to a sufficiently "... recurrent pattern of replication of bond conformations..." ${ }^{17}$ so that (a) this cannot be referred to as a "random" state, and (b) it is associated with a relatively character- 
istic CD spectrum. As we have noted explicitly, ${ }^{27}$ other environmental factors besides side chain electrostatic repulsions could give rise to similar $\varphi, \psi$ restrictions, and there is no, and never was any, contradiction in finding the $\mathrm{EH}$ type of $\mathrm{CD}$ spectrum for systems in which electrostatic repulsions are not a factor.

The above analysis provides the framework for interpreting the current results involving urea and $\mathrm{GuHCl}$ interactions with polypeptides and proteins. Blout and Fasman ${ }^{30}$ noticed that the negative optical rotation and the intrinsic viscosity of poly-L-proline in form II were increased by urea. We find an increased intensity of the positive $\mathrm{CD}$ band related to this structure in urea and of both positive and negative bands in $5.7 \mathrm{M}$ $\mathrm{GuHCl}$ (Figure 1). The conformation of the polymer in the solid state form II is known from X-ray work ${ }^{31}$ to be a $3_{1}$ helix. The same basic structure is present in solution since a similar CD spectrum is obtained from solution and from films made by casting, although the film CD is slightly red-shifted. ${ }^{32}$ A $3_{1}$ helix is a very open structure compared to an $\alpha$-helix. Urea or $\mathrm{GuHCl}$ molecules which bind to the available backbone carbonyl groups pre-empt available space and thereby reduce the flexibility of the chain. ${ }^{33}$ This would be expected to increase the intensity of CD bands. This is suggested by the work of Goodman et al., ${ }^{16}$ who found the CD bands of rigid bicyclic amides to be five times as intense as those of the corresponding monocyclic compounds, and related this to the corresponding increase in stiffness. To preserve the structure while increasing the stiffness of the $3_{1}$ helix demands that urea or $\mathrm{GuHCl}$ interact in a monovalent way, one molecule of perturbant to one carbonyl group, since bonding to more than one would distort the structure.

Our analysis of the mechanism of interaction is strengthened by a similar increase in the intensity with urea and $\mathrm{GuHCl}$ of the $\mathrm{CD}$ bands of PL and PGA in their ionized state. The conformations of these polypeptides have already been identified with a local EH structure, ${ }^{21,23}$ a form which approaches a $3_{1}$ helix. That $8 M$ urea is capable of disrupting $\alpha$-helical PL and giving in its place an EH structure is indicated by the appearance of a positive band in the $220 \mathrm{~nm}$ region. The possibility that urea breaks up aggregates of PGA molecules through a binding to side-chain carbonyl groups is suggested by the effect of urea at low $\mathrm{pH}$. This effect is followed, however, at higher concentrations of urea by another interaction which leads to a partially extended state (see Fig. 4). We believe this to be a binding of urea to the main chain carbonyl groups.

The interaction of GuHCl with ionized PL and PGA permits a possible evaluation of the differential effect of the charged $\mathrm{GuH}^{+}$ion as compared to the the uncharged urea molecule with respect to the side chain structure (cf. Figures 2, 3, and 4). As we have seen, at low concentrations of $\mathrm{GuHCl}$ the EH state is partially disrupted, an effect similar to that produced by simple salts such as $\mathrm{NaCl} .{ }^{25}$ As the concentration of $\mathrm{GuHCl}$ is increased, the CD spectra of both PL and PGA indicate a return of the EH structure, but more enhanced for PL than for PGA. The detailed reasons for this 
behavior are not as yet clear, but it is possible that the $\mathrm{COO}^{-}$groups of PGA interact with the $\mathrm{GuH}^{+}$ion, thus diminishing the extent of interaction of these ions with the backbone peptide groups in comparison with what is feasible in the case of $\mathrm{PL}$ at comparable $\mathrm{GuHCl}$ concentrations.

Incidentally, the conclusion reached by Dearborn and Wetlaufer, ${ }^{34}$ viz., that an EH state does not exist, was based in part on experiments on $\mathrm{PL}$ at only one $\mathrm{GuHCl}$ concentration, $6 M$. They apparently assumed that $\mathrm{PL}$ is in a random conformation under these conditions. As we have seen, interaction of the $\mathrm{GuHCl}$ molecule with the main chain is probably the dominant factor in determining the chain conformation in $6 M \mathrm{GuHCl}$. We therefore believe that the conclusions of Dearborn and Wetlaufer ${ }^{34}$ in this regard are unjustified.

In order to see if similar mechanisms of action of urea and $\mathrm{GuHCl}$ can be identified in proteins, we have looked at the CD spectra of many examples. To be able to extend and take an EH form requires that crosslinks either break or not interfere with extension. A high percentage of proline residues distributed along the chain would interfere with the possibility of a locally ordered repeat, and a large content of glycine residues would make a stiff structure less likely. Myoglobin, which has no S-S bonds, was found to give a positive band in the $220 \mathrm{~nm}$ region in $9 M$ urea after first heating to $95^{\circ} \mathrm{C}$ for five minutes followed by cooling to $5^{\circ} \mathrm{C}$. Ribonuclease, in spite of having four S-S bonds, is easily denatured by urea at room temperature. On cooling to $5^{\circ} \mathrm{C}$ a positive band appears in the $220 \mathrm{~nm}$ region. A similar $\mathrm{CD}$ band has been reported for the $\mathrm{S}$ peptide of ribonuclease in $5 M \mathrm{GuHCl} .{ }^{35}$ This terminal section of the ribonuclease chain has no S-S bonds nor any tyrosine or tryptophan residues. This allows the $\mathrm{CD}$ to be interpreted without ambiguity as arising from conformational changes of the backbone structure of the polypeptide. When this section is part of the ribonuclease molecule it may be expected to interact with urea and $\mathrm{GuHCl}$ in a manner similar to that of the isolated chain, and this may be the reason why a positive band is found. In lysozyme, although the overall structure of the enzyme is rather similar to ribonuclease, ${ }^{36}$ there is no long end of the molecule which is not crosslinked by S-S bonds. This may be the reason that we could not achieve a positive $220 \mathrm{~nm}$ band with urea or $\mathrm{GuHCl}$ in this case. But whereas the internal structure of the molecule is firmly resistant to urea, the exposed surface apparently can respond, as shown by the dramatic increase in intensity of the near-u.v. CD bands attributed to tryptophan, as noted by us (Fig. 9) and others. ${ }^{11,12}$ Many of the nmr lines remain broad in $9 M$ urea, ${ }^{37}$ also indicating an intact core structure. We do not agree with Shimaki et al., ${ }^{11}$ that there is a specific interaction of urea or $\mathrm{GuHCl}$ with tryptophan since we see similar CD spectral changes with such different perturbants as alcohols and denaturing salts. ${ }^{15}$ It seems more probable that the weakened surface chain interactions allow tryptophan 62 and tryptophan 63 to interact more closely. This possibility could be explored by looking for the effect of ring currents in the nmr spectra. 
We see, in general, that urea and $\mathrm{GuHCl}$ denature a protein to give a resultant state having a shoulder in the $220 \mathrm{~nm}$ region of the CD spectrum. In every case tested, this shoulder becomes more evident on lowering the temperature, a result consistent with the development of a locally ordered structure. ${ }^{27}$ (It is interesting to note that as early as 1930, Hopkins ${ }^{38}$ stated that the interaction of urea with proteins is best explained as a reversible association which is more pronounced at low temperatures.) The shape of the CD spectrum is very different from that obtained in high concentrations of $\mathrm{CaCl}_{2}$ and $\mathrm{LaCl}_{3}$ on the same proteins, where a highly unordered state is postulated by us. ${ }^{15,23}$

Tanford ${ }^{39}$ has stressed that $6 M \mathrm{GuHCl}$ randomizes most proteins. We point out, in the context of the discussion at the beginning of this section, that the structure must be thought of more in terms of a locally ordered $\mathrm{EH}$ state, with definite indication of greater order at low temperatures. The evidence for a random coil structure has been based mainly on analysis of viscosity data at room temperature. We agree with Tanford that $\mathrm{GuHCl}$ and urea lead to expanded chain structures. We believe, however, that local order can exist, especially at low temperatures, as a result of bonding of these molecules to the backbone carbonyl groups. Other evidence that has been presented for a random coil structure, such as sharp nmr signals ${ }^{40}$ and titration data, ${ }^{41}$ does not select between our locally ordered EH state and an unordered extended chain. This is because both structures freely present their side-chain groups for interaction with the solvent, and main chain flexibility exists in both cases.

Better bonding of urea and $\mathrm{GuHCl}$ to the backbone carbonyl groups at low temperatures allows a molecular explanation of the many cases of "inverse" temperature transitions, such as the partial cold denaturation of $\beta$ lactoglobulin by $5 M$ urea, ${ }^{42}$ and a similar effect of low concentrations of urea on ribonuclease. ${ }^{43}$ It would seem that at low temperatures parts of the molecule respond and interact with urea in spite of the fact that the main body of the molecule remains cross-linked. Near room temperature this interaction is weakened. Then, on raising the temperature above room temperature the cross-links can break and more of the structure can interact with the urea or $\mathrm{GuHCl}$, resulting in a normal temperature-denaturation behavior. Water should also interact by hydrogen bonding to exposed carbonyl groups. Therefore we expect that even in aqueous solution there is a temperature of maximum stability of a globular protein molecule.

There still exists the possibility that some localized free energy effects at hydrophobic side chains take part in some of the initial disruption of the native configuration. Thus, the data of Herskovits et al. ${ }^{44}$ imply that alkyl substitution and increase in temperature both decrease the total amount of denaturant needed to produce denaturation, but at the same time they found that unsubstituted urea produced a greater overall change in optical rotation. We interpret this to mean that methyl and other substituted groups on urea result in steric hindrance to binding to the backbone. 
Binding of urea and GuHCl probably occurs preferentially to the carbonyl group rather than the $\mathrm{NH}$ group. This is supported by some studies ${ }^{45,46}$ which indicate that the $\mathrm{C}=0$ group is a strong proton acceptor and the $\mathrm{NH}$ group only a weak proton donor. It may also be indicated by the generally greater effectiveness of $\mathrm{GuHCl}$ as compared to urea, which may be due to the fact that the $\mathrm{GuHCl}$ molecule presents more possible ways of interaction with the carbonyl group than does urea. This result may, however, be partly a consequence of side-chain electrostatic interactions, since in the case of the unionized water-soluble poly- $N^{5}$-(3-hydroxypropyl)-L-glutamine we have noted that urea and $\mathrm{GuHCl}$ are equally effective in producing an $\mathrm{EH}$ state.

M.L.T. wishes to thank the National Institutes of Health for support as a predoctoral trainee on PHS Training Grant GM 1355 from the National Institute of General Medical Science NIH, as well as from an NIH postdoctoral fellowship. This research was also supported by National Science Foundation Grant GB-15682.

\section{References}

1. G. Nemethy, Angew. Chem., 6, 195 (1967).

2. A. Mirsky and L. Pauling, Proc. Natl. Acad. Sci., 22, 439 (1936).

3. J. A. Schellman, Compt. Rend. Trav. Lab. Carlsberg, Ser. Chim., 29,223 (1955).

4. W. Kauzmann, Adv. Protein Chem., 14, 1 (1959).

5. P. Robinson and W. P. Jencks, J. Am. Chem. Soc., 87, 2462 (1965).

6. G. A. Vidulich, J. R. Andrade, P. P. Blanchett, and T. J. Gilligan, J. Phys. Chem., 73, 1621 (1969).

7. J. R. Warren and J. A. Gordon, Biochim. Biophys. Acta, 229, 216 (1971).

8. J. A. Gordon, Biochemistry, 11, 1862 (1972).

9. J. F. Cassim and J. T. Yang, Biochem. Biophys. Res. Commun., 26, 58 (1969).

10. E. H. Eylar and M. Thompson, Arch. Biochem. Biophys., 129, 468 (1969).

11. N. Shimaki, K. Ikeda, and K. Hamaguchi, J. Biochem. (Tokyo), 70, 497 (1971).

12. K. P. Barnes, J. R. Warren, and J. A. Gordon, J. Biol. Chem., 247, 1708 (1972).

13. K. Yutani, A. Yutani, A. Imanishi, and T. Isemura, J. Biochem. (Tokyo), 64, 449 (1968).

14. V. P. Saxena and D. B. Wetlaufer, Biochemistry, 9, 5015 (1970).

15. M. Lois Tiffany, Ph.D. Thesis, University of Michigan (1971).

16. M. Goodman, C. Toniolo, and J. Falcetta, J. Am. Chem. Soc., 91, 1816 (1969).

17. P. J. Flory, Statistical Mechanics of Chain Molecules, Interscience, New York, 1969, p. 31 .

18. D. A. Brant, W. G. Miller, and P. J. Flory, J. Mol. Biol., 23, 47 (1967).

19. G. Holzwarth and P. Doty, J. Amer. Chem. Soc., 87, 218 (1965).

20. M. Lois Tiffany and S. Krimm, Abst. Biophys. Soc. TE, 5 (1967).

21. M. Lois Tiffany and S. Krimm, Biopolymers, 6, 1379 (1968).

22. M. Lois Tiffany and S. Krimm, Biopolymers, 6, 1767 (1968).

23. M. Lois Tiffany and S. Krimm, Biopolymers, 8, 347 (1969).

24. S. Krimm and J. E. Mark, Proc. Natl. Acad. Sci., 60, 1122 (1968).

25. S. Krimm, J. E. Mark, and M. Lois Tiffany, Biopolymers, 8, 695 (1969).

26. W. B. Ripon and A. G. Walton, Biopolymers, 10, 1207 (1971).

27. M. Lois Tiffany and S. Krimm, Biopolymers, 11, 2309 (1972).

28. E. W. Ronish and S. Krimm, Biopolymers, 11, 1919 (1972).

29. W. A. Hiltner, A. J. Hopfinger, and A. G. Walton, J. Amer. Chem. Soc., 94, 4324 (1972). 
30. E. R. Blout and G. D. Fasman, Recent Advan. Gelatin and Glue Res., ed. G. Stainsby, 1, 122 (1967).

31. P. M. Cowan and S. McGavin, Nature, 176, 501 (1955).

32. M. Lois Tiffany, unpublished.

33. C. M. Venkatachalam and S. Krimm, IV International Biophysics Congress, Moscow, 1972, Abstract $E$ VI c 4/4.

34. D. G. Dearborn and D. B. Wetlaufer, Biochem. Biophys. Res. Commun., 39, 314 (1970).

35. W. A. Klee, Biochemistry, 7, 2731 (1968).

36. R. E. Dickerson and I. Geis, Structure and Action of Proteins, Harper and Row, N. Y. (1969).

37. J. H. Bradbury and N. L. R. King, Aust. J. Chem., 22, 1083 (1969).

38. F. G. Hopkins, Nature, 126, 328 (1930).

39. C. Tanford, Advan. Protein Chem., 23, 121 (1968).

40. C. C. McDonald and W. D. Phillips, J. Am. Chem. Soc., 91, 1513 (1969).

41. Y. Nozaki and C. Tanford, J. Am. Chem. Soc., 89, 736 (1967).

42. N. C. Pace and C. Tanford, Biochemistry, 7, 198 (1968).

43. J. G. Foss and J. A, Schellman, J. Phys. Chem., 63, 2007 (1959).

44. T. T. Herskovits, H. Jaillet, and A. T. Desena, J. Biol. Chem., 245, 6511 (1970).

45. C. G. Cannon, Mikrochim. Acta, 2-3, 555 (1955).

46. T. Mizushima, M. Tsuboi, T. Shimanouchi, and Y. Tsuda, Spectrochim. Acta, 7, 100 (1955).

Received June 1, 1972

Revised September 26, 1972 